\title{
A New Concept of 4D Virtual Planning in Dentistry
}

\author{
Ryakhovsky Alexandr N* and Ryakhovsky Stanislav A \\ Central Research Institute of Dental and Maxillofacial Surgery, Moscow, Russia
}

Submission: February 18, 2020; Published: March 09, 2020

*Corresponding author: Ryakhovsky Alexandr N, Central Research Institute of Dental and Maxillofacial Surgery, Moscow, Russia

\section{Abstracts}

The article is devoted to the analysis of the concepts of dental treatment planning in a traditional and digital way. Existing deficiencies are indicated. A new proposed concept of digital planning (4D) is notable for the fact that the basis for planning the next stage of treatment is not the result of the previous stage of treatment, but its virtual result. Thereby, designing occurs not only in 3D virtual space, but in time as well. The design of all stages of treatment (virtual determination of the central ratio of the jaws (positioning of the lower jaw), the movement of fragments of the jaw bones, teeth alignment, staging of artificial teeth and optimizing their shape, implantation planning) is carried out simultaneously from the initial state to the final result of treatment. The specialized functions of the software responsible for the stages of treatment interact with each other in such a way that the correction of any stage does not entail the alteration of the entire project. The predicted results of using 3D technologies to implement the planned treatment are guaranteed to achieve the desired goals. When using the proposed concept, a reduction in the probability of an error due to the human factor is expected, as well as a reduction in the treatment time for patients, since different types of the treatment can be performed simultaneously. The article provides examples of the digital treatment planning in the 4D concept

\section{Introduction}

In recent years, digital technologies have been actively penetrating all areas of human life, providing a high level of convenience and efficiency. Dentistry is no exception. At the same time, 3D technologies, as a part of digital dentistry, enjoy rapid development $[1,2]$. Computer and magnetic resonance imaging provide the highest level of diagnostics [3]. Cad/Cam technologies for manufacturing of dental prostheses provide a consistently high level of quality, expand the range of construction materials used, and reduce costs $[4,5]$. 3D modeling for planning clinical results of treatment and its achievement based on 3D technologies is still used quite rarely, partly and step by step. There are various software products capable to plan in 3D certain types of treatment, for example, teeth alignment, implant navigation, smile design [6]. The advantage of using them is a high probability of achieving virtually planned result, the disadvantage is the impossibility of complex application. The use of 3D technologies in such case is a key to achieving a planned result, but the planning itself does not much differ from the traditional approach. Traditional planning is based on the concept of sequential step-by-step planning (Figure 1). Its main drawback is that it is not possible to plan immediately the final result of a multi-stage treatment and show it to the patient. Planning is performed sequentially before each new stage of the treatment, and the initial data for it are the results of the previous stage (Figure 1).

At the same time, each new stage is planned not by one, but by different specialists and their ideas of the final result may differ. Such a planning assumes that these specialists have a similar idea of the desired result and how to achieve it. Teamwork is crucial in this concept. The lack of expertise and competent planning at any stage can has an adverse impact on the overall result, and there is a high probability of gradual deviation from the ideal result at the stages. Theoretically, such an approach allows planning the final result, provided that the data format is open, and data are transferred from one software to another. However, in real life, this is not feasible because of the closed data format of different types of software, a trite inconvenience and additional time losses. It is also important that the user must learn first all the features of different types of software. At the moment, there are only few software products, which have necessary functionality on a single platform and allow to implement the concept of sequential final planning (Figure 2).

This concept is based on sequential planning of treatment stages to the final result. A high probability of achieving the planned result is possible by using 3D technologies: CAD $\backslash \mathrm{CAM}$ 


\section{Advances in Dentistry \& Oral Health}

technologies repeat the shape of virtual artificial teeth with a high accuracy in real products, surgical guides place implants to the planned position, and previously planned alignment of teeth is also achieved with high probability using 3D technologies. The difference and main advantage of this concept is that not the result of treatment, but the result of virtual planning at the previous stage can be the basis for further planning. Thus, this concept already contains elements of 4D modeling since organizes not only the position and shape of 3D objects itself, but also their changes over stages of planned treatment (time). The main disadvantage of this approach is that if an error was made at the previous stage or a small correction is required, then it is necessary to redo it and all subsequent stages (Figure 3).

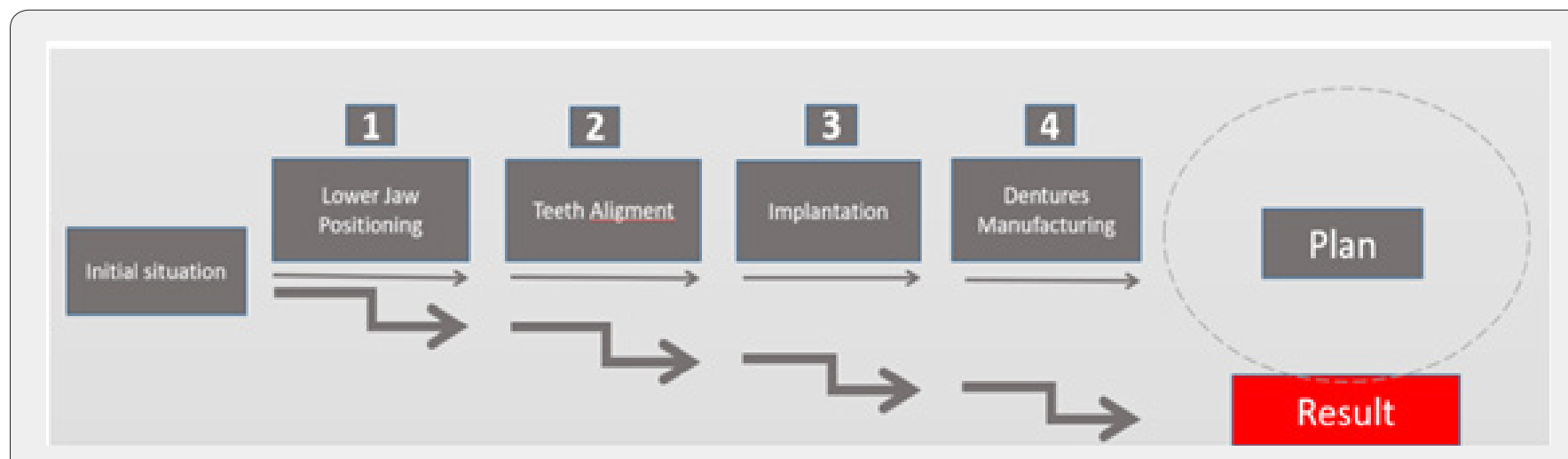

Figure 1: Traditional sequential step-by-step planning scheme.

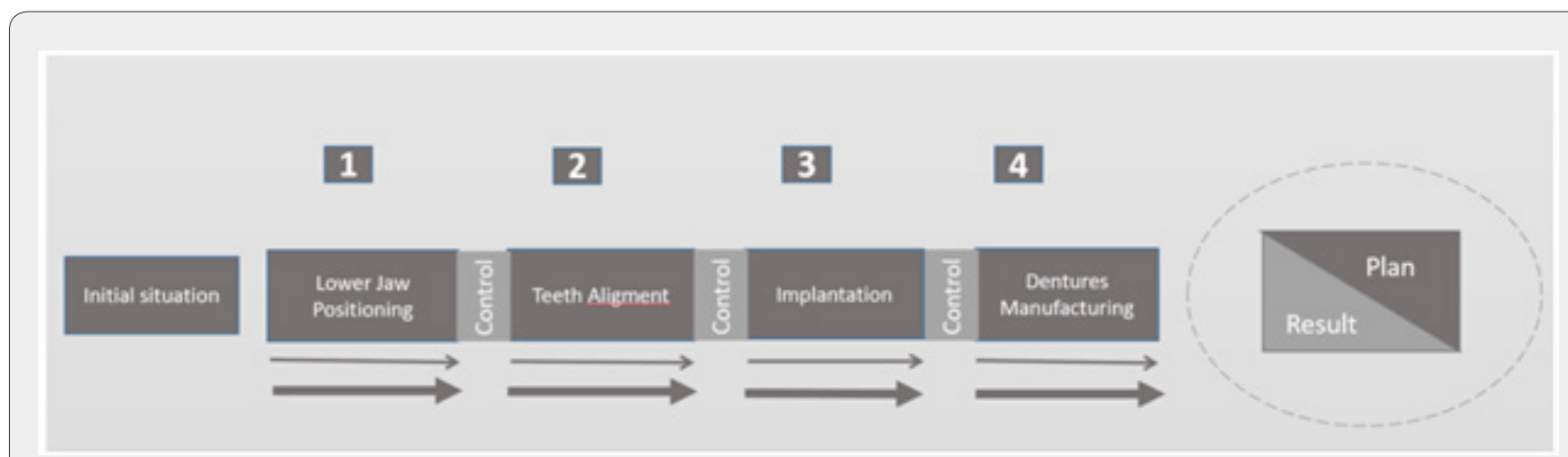

Figure 2: The sequential final planning scheme.

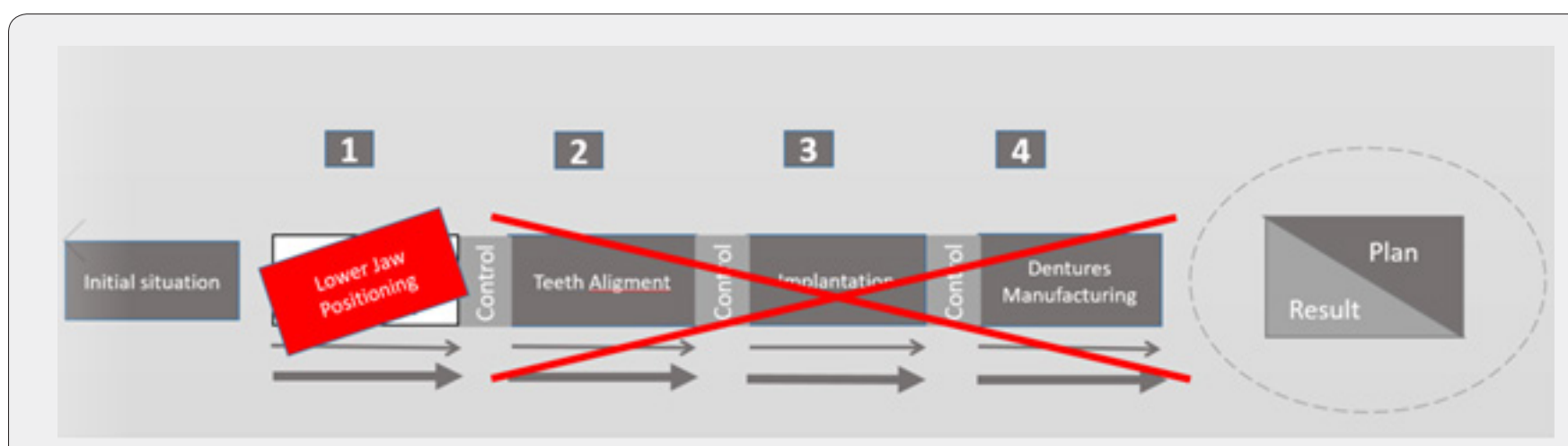

Figure 3: Correction of one of the intermediate stage's "breaks" the entire modeling project and requires its complete rework..

For example, if the central relation of lower jaw is set virtually, and then a teeth alignment scenario is created, and it turns out that occlusal contacts could become more optimal if to correct a little bit this position of a lower jaw, it is required to redo all the work 
from the very beginning. Or, if the expected by the patient result differs from the one created by the dentist? Such situations are very common on planning the result of complex dental treatment with more than one stage. Since virtual 3D planning still requires a significant amount of time, this disadvantage becomes very essential and in real practice insurmountable.

In this regard, the great potential of such software products that have full functionality on a single platform cannot be fully applied, and user perform traditional sequential step-by-step planning. The only difference is that is more convenient to use only one software product then a few others. We propose a new concept of 4D modeling (Patent Cooperation Treaty, PCT/ $\mathrm{RU} / 2019 / 050208$ ). Instead of step-by-step planning, it is based on one-step (simultaneous) final planning. Its peculiarity is that all the functionality of the clinical 3D modeling (mandible positioning/moving dento-alveolar fragments, teeth alignment, artificial teeth arrangement, implant planning) must be combined in a single unit and work in such a way that the planning of different interrelated stages of treatment should be performed prior to the treatment, include all the necessary stages, with the possibility to correct any stage, and this correction automatically or manually leads to a corresponding adjustment of all the others (Figure 4).

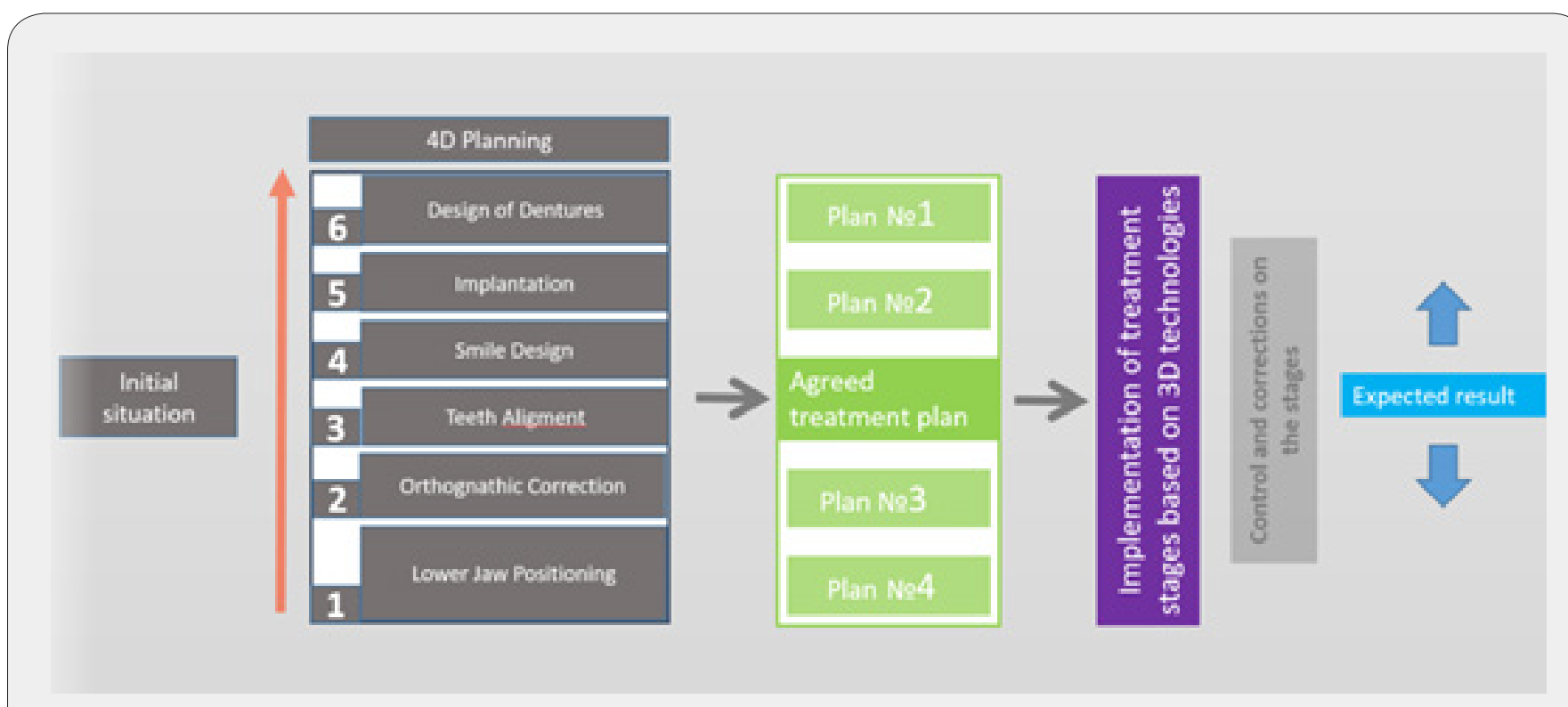

Figure 4: One-stage final 4D planning scheme

It is necessary to consider the possibility of creating several alternative treatment plans. Moreover, for each LJ central relation, for example, it should be possible to create several scenarios of teeth alignment, and for each scenario of teeth alignment, it should be possible to make different plans for artificial teeth arrangement. To implement this approach, it is necessary to provide completely unique ways of interaction between the functional modules of the software. In the computer software, among the saved objects (CT data, dental row scans, TMJ elements, mandibular canals, UJ and LJ bone, roots and crown parts of teeth, photos of the patient's smile (or scans of his face), CT cross sections in the area of interest, a specific object must be saved - the scenario of the treatment plan. This scenario should store information (or a link to information) about the specified position of the LJ (habitual occlusion or central relation), the coordinates of the transferred fragments of the jawbone (if an orthognathic surgery is planned), the scenario of teeth alignment (if there are deformities of the dentition, abnormally located teeth), the shape and position of artificial teeth and implants (if there are defects in the dentition). A complex prosthetic treatment can be performed either in habitual occlusion, or in centric relation (reconstructive, treatment position).

The position of the $\mathrm{LJ}$ is a kind of Foundation for the entire upcoming "superstructure". it is selected and set based on the analysis of the ratio of the elements of the TMJ (condyle and articular fossa) and the height of the bite. It should be possible for the user to specify several alternative options for central relation. In case of disproportionate development of the jaws and the need to perform orthognathic surgery (moving the dentoalveolar fragments of the jaws), the treatment plan may include one or more scenarios of appropriate transformations. A complex treatment plan may include creating scenario for teeth alignment, correction of the natural teeth shape (with or without correcting their position), substitution of partial adentia with artificial teeth. Information about all that should be saved in corresponding treatment plans. After that the position and the size of the implants is planned, taking into account the position of the artificial teeth, the volume of the bone, and the location of the neighboring roots. Information about that is saved as well. Here are some specific examples of such original solutions, providing complex virtual treatment planning. 


\section{Advances in Dentistry \& Oral Health}

Example 1: Simulation of Registration and Moving a part of the volumetric data (CT data)

In computer $3 \mathrm{D}$ modeling, sometimes there are situations when it is necessary to change the position of the lower jaw together with the volumetric data (for example, when LJ moves from habitual position to central relation or virtual mouth opening or closing). In the traditional approach, the patient is forced to repeat $\mathrm{CT}$ in the desired position of the lower jaw. Our approach is based on an algorithm of sequential steps of 3D modeling: a) receiving of the mesh from volumetric CT data, according to the chosen optical density; b) separation of the mesh into 2 parts: part 1 - CT-UJ (upper jaw) and part 2 - CT-LJ (lower jaw); C) registration of LJ and UJ scans, in habitual occlusion with the mesh from CT; d) registration of the mesh CT-LJ with LJ scan. At the final step, those voxels of volumetric data, that are located near and inside the perimeter of the CT-LJ mesh, moves along with it (Figure 5). Another solution is to store the matrix of such a transformation and to apply it to all subsequent virtual transformations if necessary. This variant does not assume changing voxels position in the virtual space, but only the movement of CT cross section. This approach eliminates the need to make repeated $\mathrm{x}$-rays, when, for example, it is necessary to assess the position of the condyles when changing LJ position, or to plan implantation in virtually reconstructive bite. This method can be also used for a virtual simulation of the mouth opening. It is needed for implant planning to verify if there is enough space to set hand peace with a drill in starting point position (Figure 6).

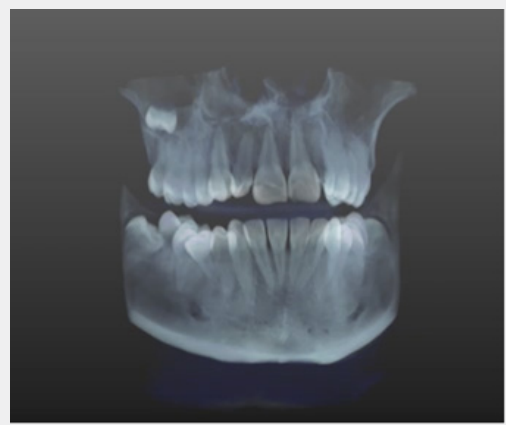

Figure 5a: The Original CT scan made with separated jaws.

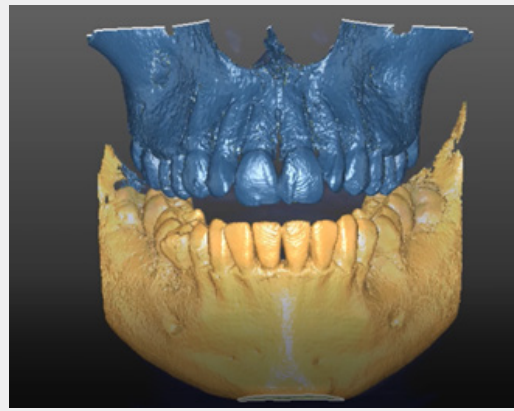

Figure 5b: Bone meshes obtained from CT data.

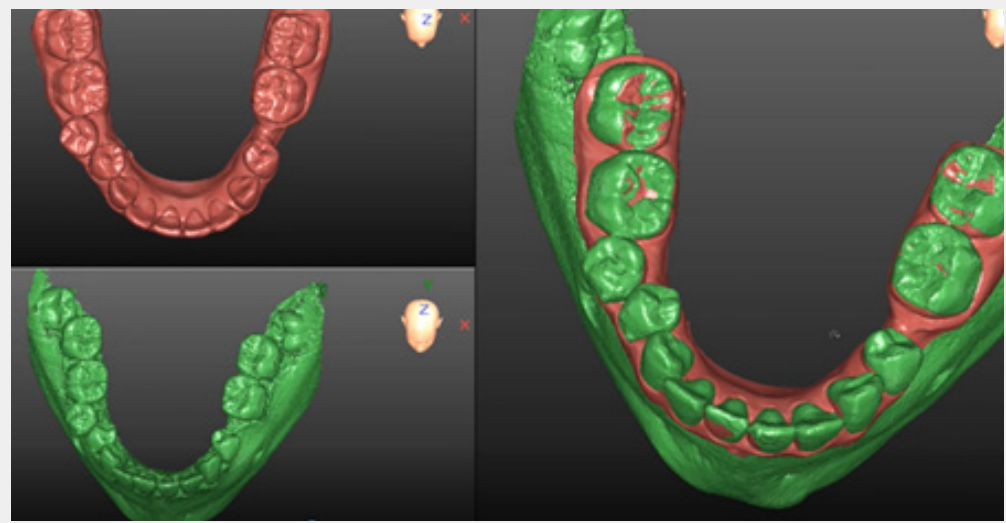

Figure 5c: CT-LJ mesh registration with a LJ scan. 


\section{Advances in Dentistry \& Oral Health}

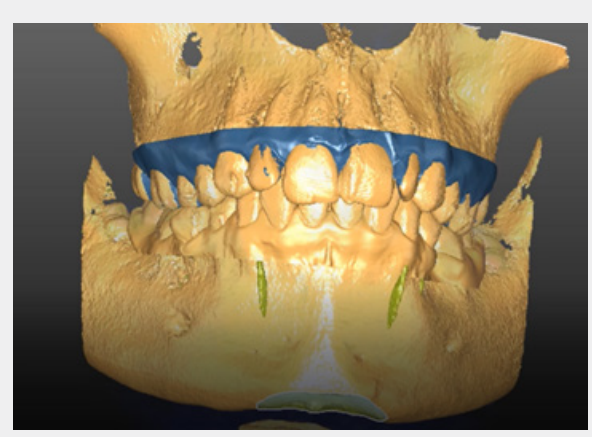

Figure 5d: Registered scans and CT meshes in the position of habitual occlusion.

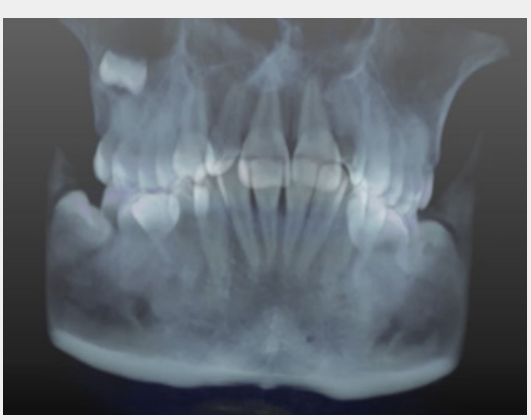

Figure 5e: Volumetric data, corresponding to the lower jaw, are transferred and stored in accordance with the matrix of registration transformation.

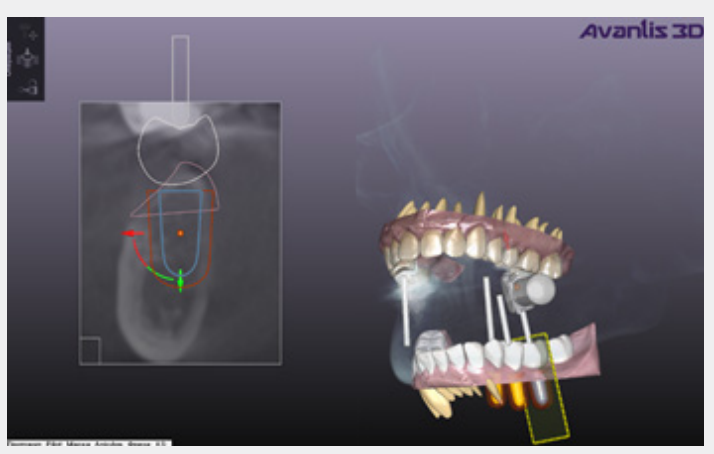

Figure 6: Simulation of the mouth opening is possible only if the 3D scene contains TMJ elements selected from CT (see Example 2).

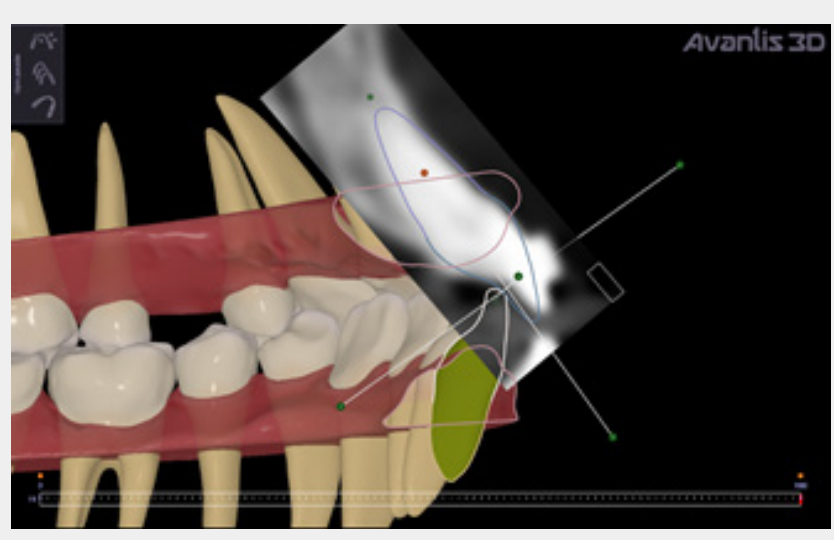

Figure 7: Control of root movement by CT cross-section. 


\section{Advances in Dentistry \& Oral Health}

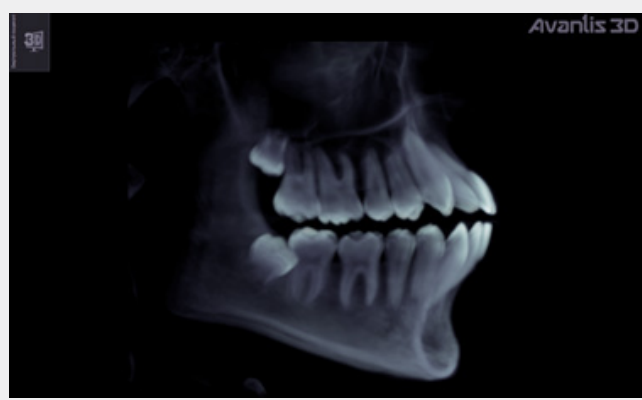

Figure 8a: Teeth are separated on CT.

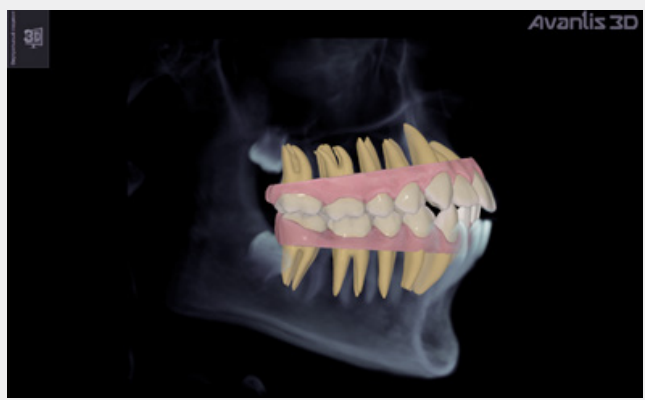

Figure $\mathbf{8 b}$ : Teeth scans in occlusion are registered with CT.

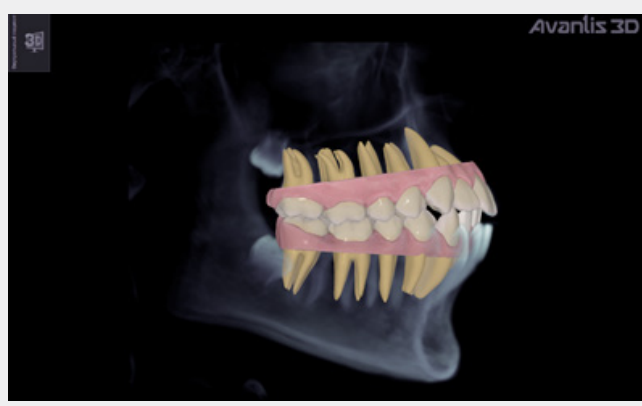

Figure 8b: Teeth scans in occlusion are registered with CT.

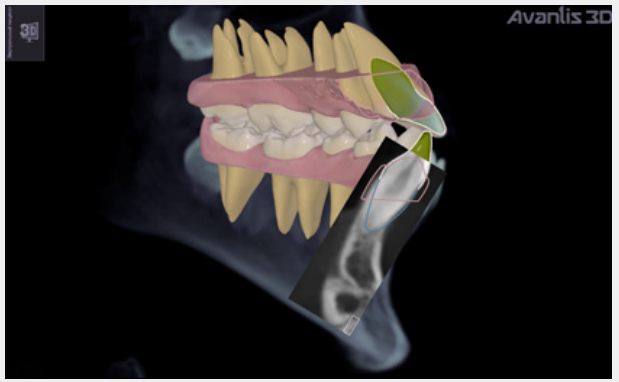

Figure 8c: CT crossection is automaticaly transferred.

In this case, the slope and the selected length of the drill are planned so that the hand peace does not touch the antagonists. The implant position is planned according the position of the virtually placed artificial teeth, the bone volume, the position of adjacent teeth, their roots, the mandibular and the incisor canals. When planning teeth alignment, a cross section shows a root position in the bone (Figure 7). In the case when CT is made with LJ in habitual occlusion, and teeth alignment is also planned in habitual occlusion, it will not cause any problems. However, if teeth alignment is planned in central relation, then the meshes (roots, crowns, etc.) and the contours of the same objects on the CT will correspond completely for the Upper Jaw, and totally differs for 
the Lower Jaw. In this regard, we use the transfer of the CT data (cross-sectional CT) in exact accordance with the transformation maposition from the habitual occlusion to central relation. We use the same principle of transferring volumetric data in cases where CT is done with separation of the dentition (arbitrary position), and the alignment of the teeth or any other treatment planning is performed in the definite position (habitual occlusion or centric relation) (Figure 8).

\section{Example 2: Virtual positioning of lower jaw to central relation}

In a complex prosthetic rehabilitation, an accurate determination of the central relation of the jaws is of critical importance. One of the criteria for the correct position is the ratio of the TMJ elements (the size of the posterior, the upper and the anterior parts of the articular gap). For this position, the values of the joint gap width norm are determined [7]. In the traditional approach, the central relation is determined clinically and fixed by occlusal bites. Its correctness can be controlled only by repeated
TMJ x-rays which require an additional irradiation of the patient. We propose controlling and setting virtually the position of lower jaw (Patent Cooperation Treaty, PCT/RU/2018/050054). This can be done by using a special algorithm:

a) Using $\mathrm{CT}$ data of the patient in habitual occlusion (maximum multiple occlusal contact of teeth), left and right articular fossae and condyles are separated.

b) Scans of upper and lower dentition are registered with CT data.

c) Virtual positioning of LJ is executed taking in to account the position of the condyles in particular fossae and the height of the bite (Figure 9). The software automatically controls the size of the gap.

Similarly, it is possible to set/correct virtually not only the position of the central relation, but also the terminal LJ positions as well: protrusion, right and left laterotrusions.

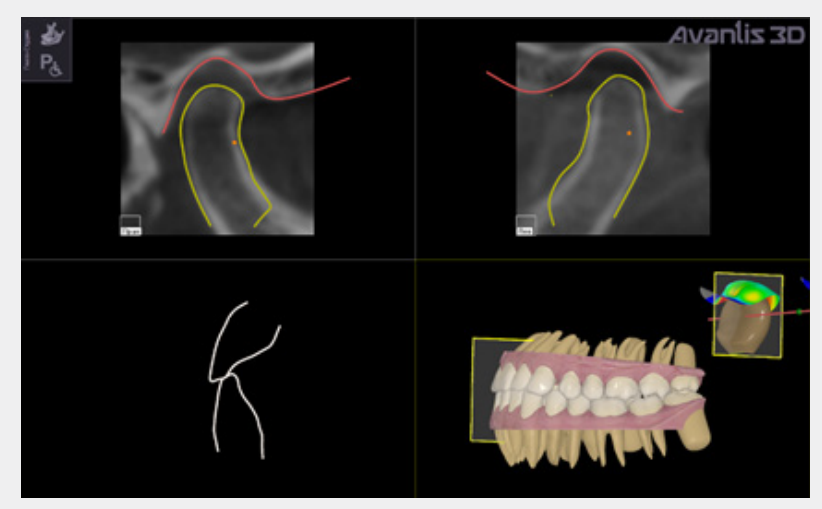

Figure 9a: Initial LJ position.

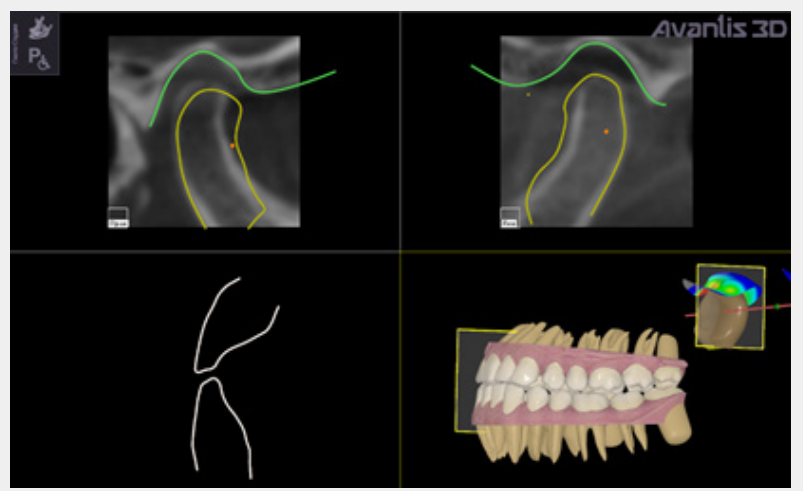

Figure 9b: The result of automatic LJ positioning in central relation.

Example 3: Planning teeth alignment when changing the lower jaw position

In clinical practice TMJ pathology is often associated with malocclusion. The problem often arises on planning teeth alignment in a new virtual LJ position. In the traditional approach the height of the bite is adjusted by using occlusal pads. But it is almost beyond the control of the LJ position in the horizontal plane. 


\section{Advances in Dentistry \& Oral Health}

When using modern computer technologies, the LJ position is set clinically first, then this position is transferred to the 3D scene and the teeth alignment is planned. But if it turns out that LJ position is set incorrectly, or it requires a slight correction for a better occlusion, it becomes clear that all the work must be redone. That is extremely inconvenient and time-consuming. We propose a mutually conditional virtual planning, when it is possible to correct any stage of the planning with automatic or manual corrections of the following stages without destroying the final result (Figure 10). An important feature is that the final position of the teeth is automatically hold when correcting LJ position. The scenario of the tooth movement is modified by changing starting position of the teeth, and their final position remains.

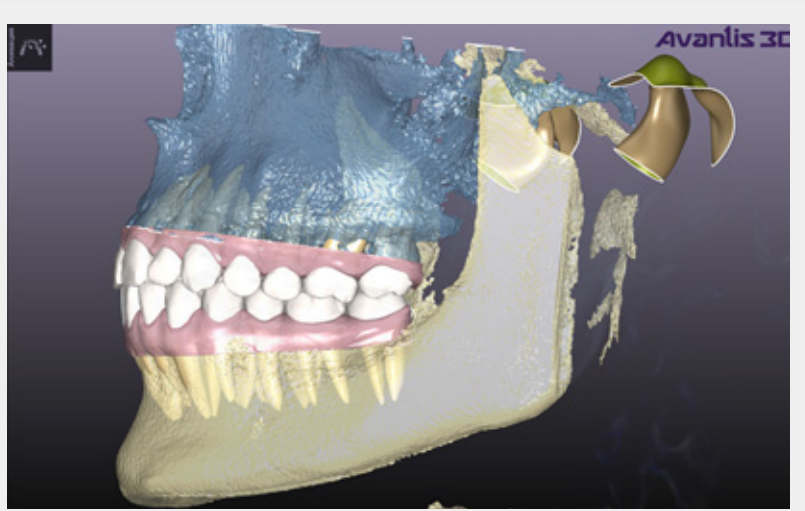

Figure 10a: Initial position of the teeth and LJ in habitual occlusion (patient has a dysfunction of temporomandibular joint after orthodontic treatment).

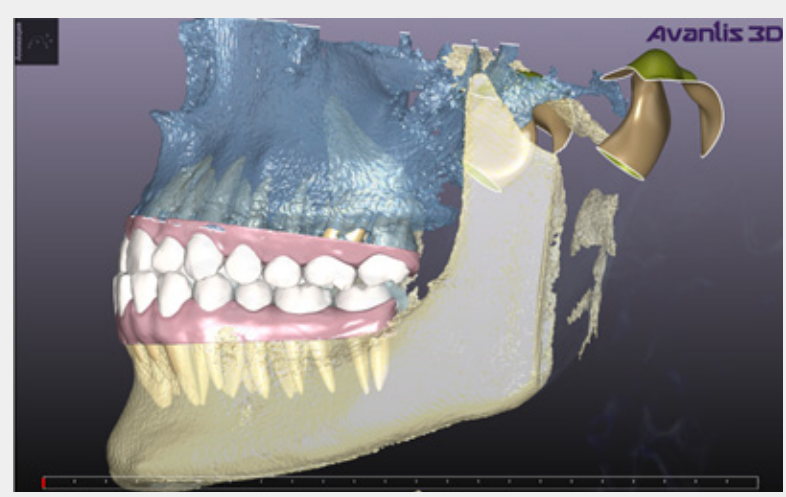

Figure 10b: LJ is transferred to central relation.

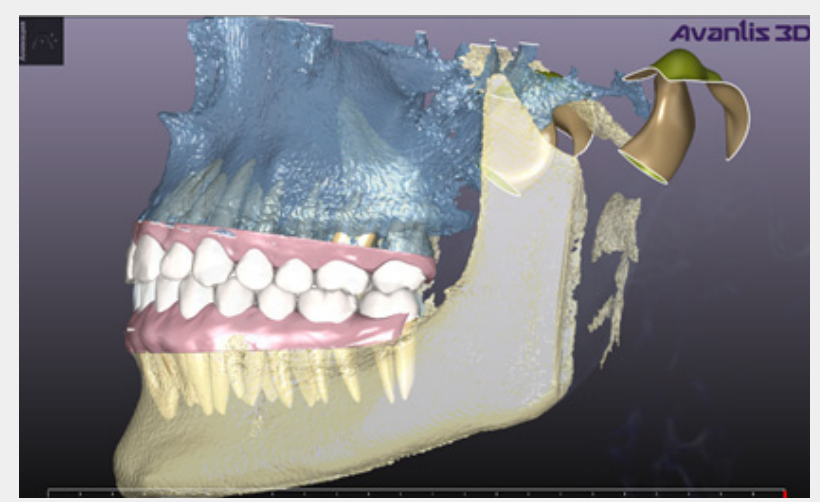

Figure 10c: Virtual set up of the teeth in reconstructive occlusion.

Example 4: Planning the position and the shape of artificial teeth when changing lower jaw position.

Not often than less, it is necessary to reconstruct the bite with fixed dentures. In traditional clinical practice, the reconstructive position of a LJ is determined first, and fixed with bites. After that, dental technician makes a new design of occlusion by wax up. In case of changing a LJ position, a new wax up should be done again. When using modern computer technologies, the position of a LJ 
is set clinically, and then transferred to the 3D scene. After that, the occlusion of the future prosthetic dentures is designed. If it turns out that, a LJ position is set incorrectly, or it requires just a slight correction, a new occlusion should be reconstructed once again. We propose mutually conditional virtual planning, when after the LJ positioning, the shape of artificial teeth is simulated with the possibility of moving to the previous stage and back for mutual corrections (Figure 11). At the same time, the import of artificial teeth from the library into the 3D scene is carried out with automatic "binding" to the necks of the natural teeth. This provides a unique opportunity for a virtual correction of the LJ position, preserving existing occlusal contacts owing to the automatic correction of the "binding" artificial teeth to the necks of natural teeth.
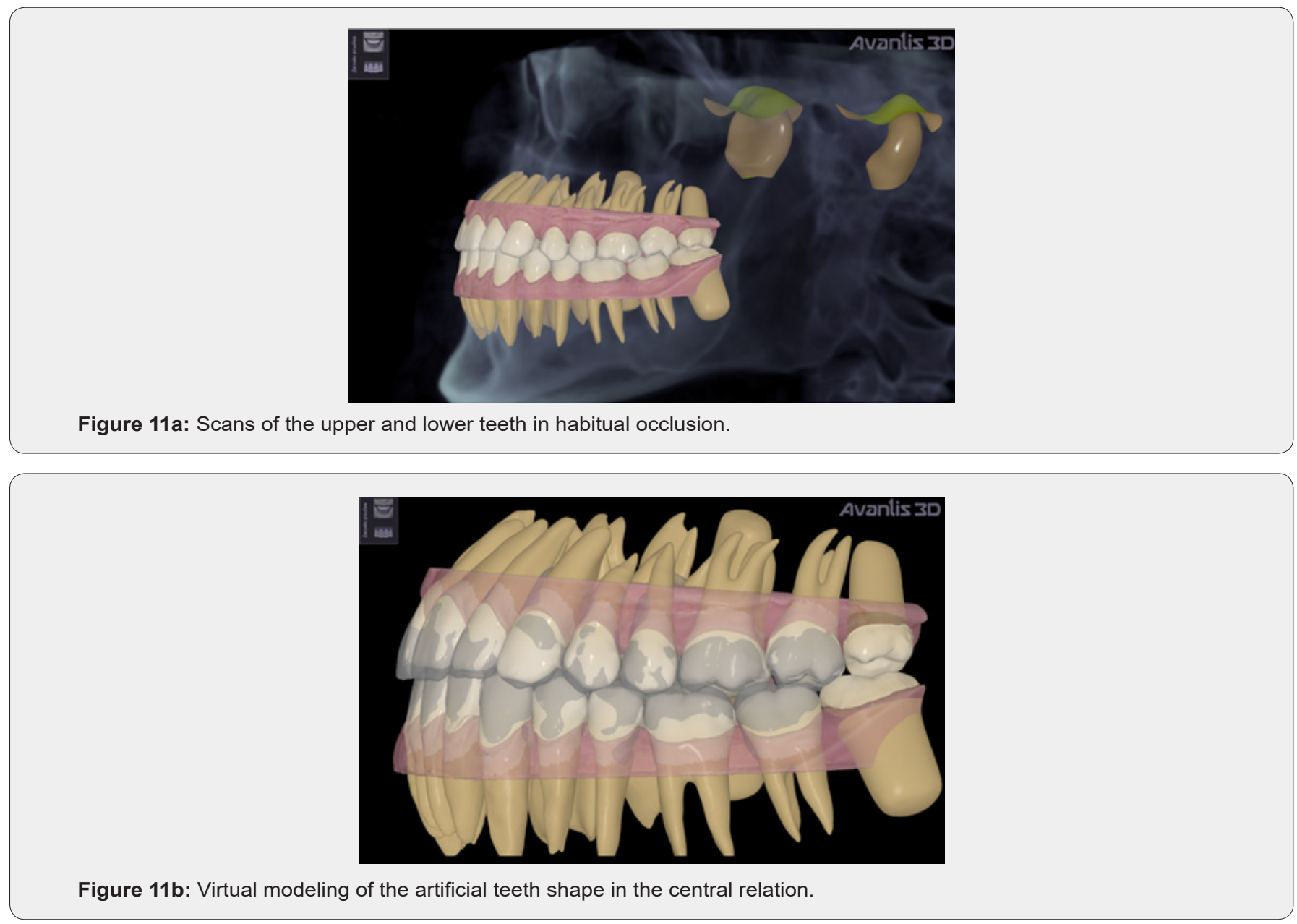

Example 4: Planning the shape of artificial teeth (on lays or crowns) when changing the position of the supporting teeth (teeth alignment)

The prosthetic dentist is most frequently faced with the task of correcting the shape or the color of the front teeth that have an incorrect position (vestibular tilt, crowded position, rotation of the teeth, etc.). In such cases, the doctor either performs excessive grinding of the teeth, or performs a preliminary orthodontic correction of their position before prosthetics. In the latter case, a more progressive concept of minimally invasive prosthetics is implemented. With the traditional approach, after correction of the teeth position, dental impressions are taken, and a new shape of the teeth is waxed. Then it is checked in the mouth by Mockup. When using computer-aided design, a virtual natural teeth alignment is performed, and then artificial teeth are placed in the final position of natural ones. With this approach, when it is necessary to correct the alignment scenario of the teeth, it generates the need of artificial teeth correction in their position, proportions and shape. Our approach is to place artificial teeth automatically, "binding" to natural ones. In this case, artificial teeth are automatically scaled based on the total width of the natural teeth being replaced and preserving the natural proportions. In addition, each artificial tooth is registered with its natural analog on the principle of the maximal correspondence, that is, the total distance between their surfaces becomes minimal, which is the key to the minimum possible subsequent preparation. In cases where there is a need to correct the scenario of teeth alignment, natural and artificial teeth move simultaneously, but the analysis 
of occlusal and approximal is conducted doing only for artificial teeth. In this case, natural teeth become "guided" and their position is subordinated to the final aesthetic or the functional result of artificial teeth (Figure 12). On the contrary, in some cases, when the first attempt for a virtual alignment was not optimal, but artificial teeth were placed in more correct position, it should be possible, when going back to the stage of the virtual alignment planning, to fix the position of the artificial teeth and to adjust the final position of the natural teeth to them [8].

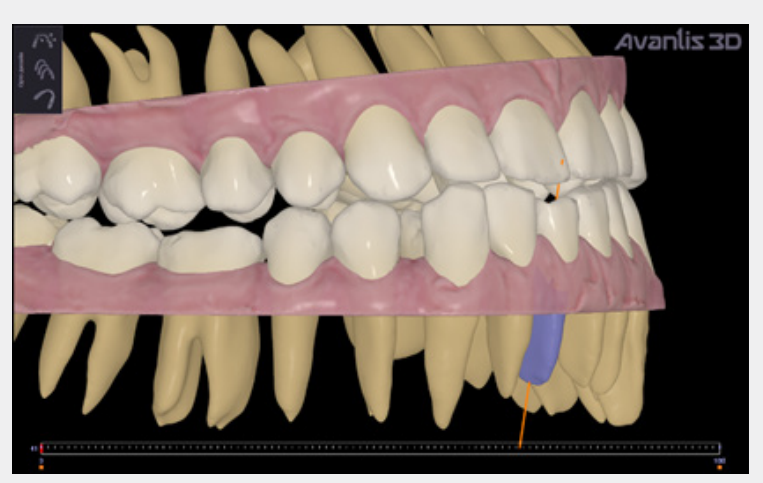

Figure 12a: Natural teeth before virtual alignment.

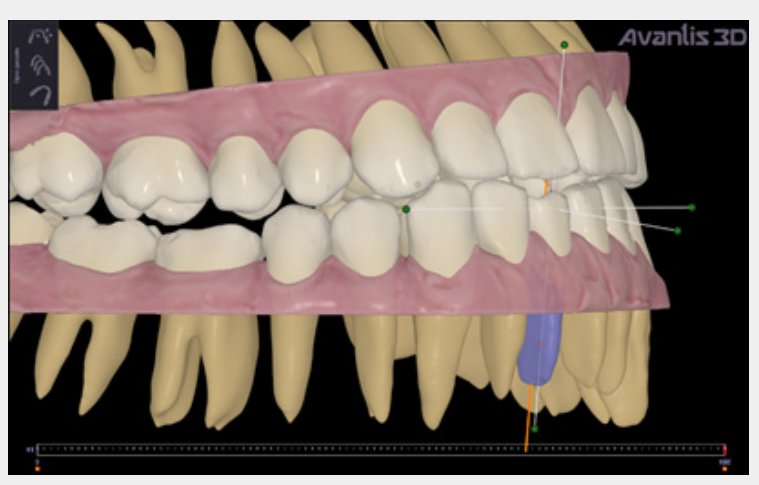

Figure 12b: Natural teeth after virtual alignment.

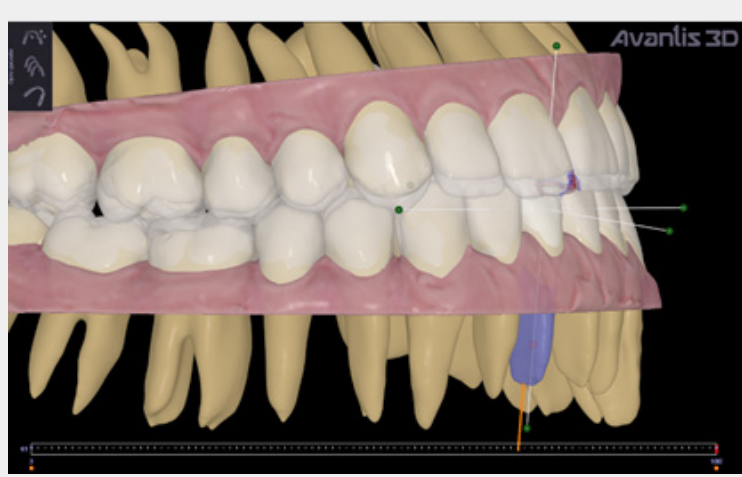

Figure 12c: Artificial teeth over natural in their final position.

Example 5: Planning the position and the shape of teeth when changing the position of jawbone fragments

Orthognathic surgery is recommended for cases with severe macro or micrognathia. Cephalometric analysis determines the amount of necessary correction, the surgery is performed, and then proceed to the stage of the orthodontic and probably the subsequent prosthetic treatment. The success of such a treatment is highly depend on the accuracy of the first stage - the orthognatic surgery. Usually virtual planning of the orthognatic surgery does not take into account the LJ position. As a result - a high probability of the TMJ dysfunction. We propose planning the movement of the jaw fragments after virtually LJ positioning with simultaneously planning teeth alignment and shape of artificial teeth (if necessary). With such a planning, the surgeon definitely 


\section{Advances in Dentistry \& Oral Health}

has a certain idea of the position in which the moving fragments should be placed in order to achieve a "perfect" bite. In accordance with this position, an occlusion splint can be designed to facilitate the positioning of the jaw fragments during surgery. In contrast to the known approach, our proposal is based on a very specific virtual planning that visualizes the final result, considering possible subsequent changes of the LJ position and the position and the shape of the teeth (Figure 13).

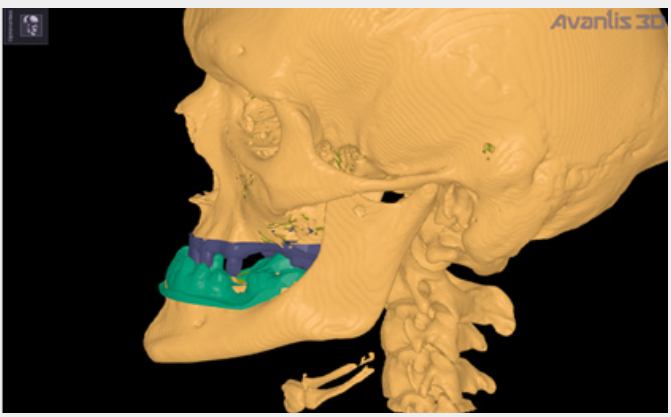

Figure 13a: Initial situation.

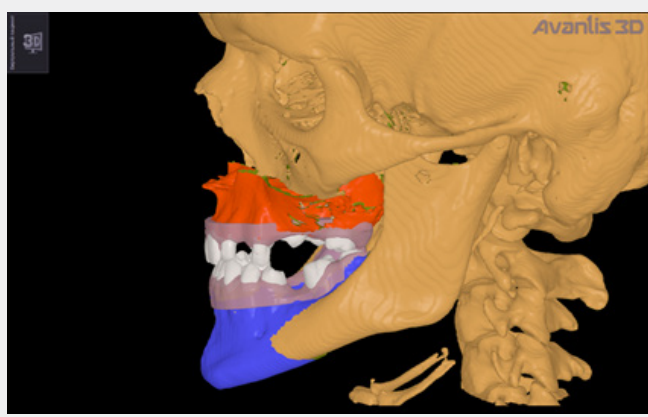

Figure 13b: Bone fragments virtual displacement.

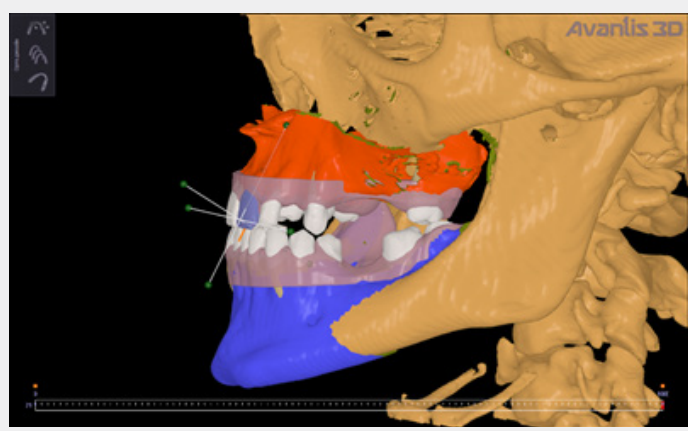

Figure 13c: Teeth alignment.

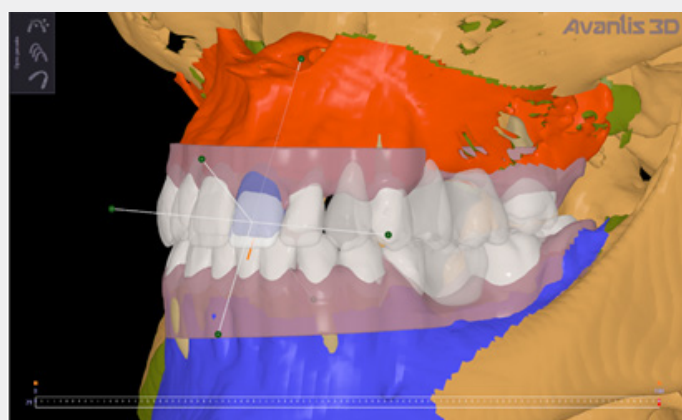

Figure 13d: Artificial teeth set up. 


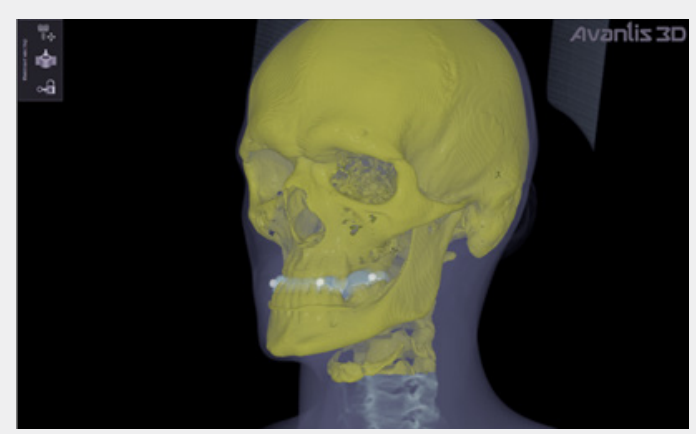

Figure 13e: Before virtual planning.

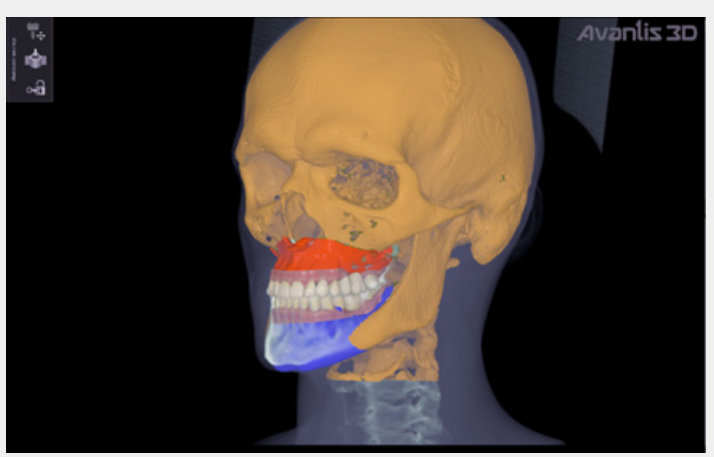

Figure 13f: The result of virtual treatment plan.

\section{Example 6: Implant planning according the position of artificial teeth}

Planning implantation according to the position of artificial teeth is not a brand-new idea in dentistry [9]. However, implant planning in 4D concept can be made with respect to the virtual a predetermined lower jaw position, displacement of teeth-alveolar complexes (fragments of the jaw) and teeth alignment for optimal final aesthetic and functional results (Figure 14).

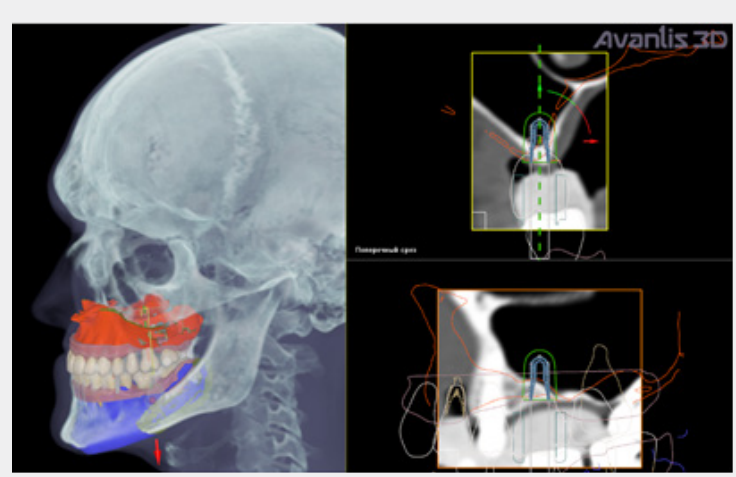

Figure 14: Implant planning after bone fragments virtual displacement, teeth alignment, smile and occlusion design.

\section{Conclusion}

The proposed concept of computer planning can be qualified as $4 \mathrm{D}$ planning, since it is performed not only in 3D virtual space, but in virtual time. With this approach, you can predict not only the final, but all the intermediate results that will be expected to be achieved at known intervals. This method of planning allows changing the traditional scheme of step-by-step treatment. Considering the high predictability of achieving results based on the use of 3D technologies, using 4D planning, dentist can start different types of treatment at the same time, thereby significantly reducing its overall duration. It makes possible simultaneously set the reconstructive lower jaw position and align the teeth; align the teeth and perform implantation, etc. there can be any quantity of combined examples. As a result, any quantity of treatment plans can be created in a relatively short period of time. Since the current level of 3D technology in most cases is not able to meet the 
high requirements for precise fitting of permanent fixed dentures, then, as a rule, the stage of permanent dentures is taken out in a separate stage and performed as the final one. This, in principle, does not go beyond the proposed concept (Figure 4) and can be considered as a correction stage. Even nowadays in some cases it is possible to make permanent dentures before the one stage of treatment - implantation.

Future development of 3D technologies makes it possible to make them before more stages and therefore to shorten treatment even more. It should be emphasized that any of the stages of treatment, due to the upcoming complexity, can be placed in a separate one, and all subsequent stages of treatment can be adjusted according to its outcome. In any case, such a decision can be justified and made at the stage of planning. Further improvement of 3D technologies in treatment will inevitably lead to an even greater probability of achieving the planned result and will allow more and more simultaneous use of combined forms of treatment (gnatological, orthodontic, surgical and prosthetic). Improving the cost-effectiveness of dental treatment by reducing its overall duration and patient visits increases the doctors' productivity and improves the quality of patients' life. They will be able to spend more time on their work or leisure. The economic feasibility of implementing the developed principles of computer 4D planning seems to be timely and appropriate.

\section{References}

1. Paul L, Child JR (2011) Digital dentistry. Is this the future of dentistry? Dent Econ 101: 10.

2. Van Noort R (2012) The future of dental devices is digital. Dent Mater 28(1): 3-12.

3. Eberhard L, Rohde S, Giannakopoulos N, Schmitter M (2013) Temporomandibular Joint (TMJ) Disk Position in Patients with TM] Pain assessed by coronal MRI. Dento maxillofacial Radiology 42(6).

4. Lee W, Lee D, Lee K (2017) Evaluation of internal fit of interim crown fabricated with CAD/CAM milling and 3D printing system. J Adv Prosthodont 9(4): 265-270.

5. Wook-Tae Kim (2018) Accuracy of dental models fabricated by CAD/ CAM milling method and 3D printing method. J Oral Res 7(4):127-133.

6. CAD/CAM dentistry | Planmeca.

7. Semkin VA, Rabukhina NA (2000) Dysfunktsia visochnonighnechelustnych sustavov (klinika, diagnostika, lechenie)-M, "Novoye v Stomatologii" 56s.

8. Samir AHC, Federico HA (2012) 3D planning in orthognathic surgery: $\mathrm{CAD} / \mathrm{CAM}$ surgical splints and prediction of the soft and hard tissues results - our experience in 16 cases. J Craniomaxillofac Surg 40(2): 162-168.

9. Widmann G, Bale RJ (2006) Accuracy in Computer-Aided Implant Surgery-A review. Int J Oral Maxillofac. Implants 21(2): 305-313.

\begin{tabular}{l} 
Your next submission with Juniper Publishers \\
will reach you the below assets \\
- Quality Editorial service \\
- Swift Peer Review \\
- Reprints availability \\
- E-prints Service \\
- Manuscript Podcast for convenient understanding \\
- Global attainment for your research \\
- Manuscript accessibility in different formats \\
( Pdf, E-pub, Full Text, Audio) \\
- Unceasing customer service \\
Track the below URL for one-step submission \\
https://juniperpublishers.com/online-submission.php \\
\hline
\end{tabular}

